\title{
Evaluación de la Patogenicidad Inhalatoria del Micoinsecticida Beauveria bassiana LF14 en Ratones
}

\author{
Assessing the Inhalation Pathogenicity of the Mycoinsecticide Beauveria \\ bassiana LF14 IN MICE
}

\author{
Dalmiro Cazorla Perfetti ${ }^{1,2}$, María Acosta Quintero', Pedro Morales Moreno ${ }^{1}$
}

\section{Resumen}

Se realizaron pruebas de bioseguridad para evaluar los posibles efectos patogénicos del aislamiento autóctono LF14 de Beauveria bassiana en ratones albinos NMRI; el cual posee una elevada virulencia hacia Rhodnius prolixus y Triatoma maculata (Triatominae), vectores de enfermedad de Chagas en Venezuela. Se administró 1×109 conidias/ml del hongo mediante gotas nasales. Se realizaron observaciones clínicas y de comportamiento, así como de peso corporal en forma diaria y por 14 días. Asimismo, se estimó el aclaramiento fúngico mediante examen directo y cultivo de muestras intranasales, y de infectividad con la toma de muestras de tejidos de varios órganos, incluyendo un estudio histopatológico a los 3, 7 y 14 días después de la inoculación. No ocurrieron muertes ni alteraciones clínico-patológicas discernibles, siendo el tipo de comportamiento activonormal en el $100 \%$ de los casos. El peso promedio corporal se incrementó, tanto en los animales expuestos a LF14 B. bassiana como los controles, habiendo diferencias entre $\operatorname{sexos}(\mathrm{p}<0.0000)$. No se detectaron anormalidades en la inspección anatomopatológica ni hubo reacciones tisulares de consideración. Solo se obtuvieron conidias viables sin germinar al día tres de la necropsia en pulmón y tráquea, sugiriendo que no existe evidencia de multiplicación fúngica. Los resultados obtenidos permiten sugerir que el aislamiento B. bassiana LF14 no es patogénico por vía inhalatoria en Mus musculus (NMRI).

Palabras clave: Beauveria bassiana, patogenicidad, ratones, infectividad

\section{Abstract}

Biosafety tests were conducted on albino mice (NMRI strain) to evaluate pathogenic effects of a native isolate LF14 of B. bassiana, highly virulent to Rhodnius prolixus and Triatoma maculata (Triatominae), vectors of Chagas disease in Venezuela. A total of

\footnotetext{
${ }^{1}$ Laboratorio de Entomología, Parasitología y Medicina Tropical (L.E.P.A.M.E.T.), Centro de Investigaciones Biomédicas (C.I.B.), Universidad Nacional Experimental «Francisco de Miranda»(UNEFM), Estado Falcón, Venezuela

${ }^{2}$ E-mail: lutzomyia@hotmail.com

Recibido: 4 de marzo de 2015

Aceptado para publicación: 10 de junio de 2015
} 
$1 \times 10^{9}$ conidias $/ \mathrm{ml}$ of the fungus was administered to the animals by nasal drops. Body weight control and clinical and behavioural evaluations were done daily. Likewise, clearance of fungus was estimated by direct examination and culturing of intranasal samples, and infectivity by performing mycological and histopathological test after necropsies at 3, 7 and 14 days post-inoculation. Animals no showed discernible pathological changes or death and all had active-normal behaviour. Body weight increased in both treated and control mice groups, with statistically difference between sexes $(\mathrm{p}<0.0000)$. Anatomopathological changes were not detected. Only was observed viable but nongerminated conidia at day 3 in lung and traquea tissues, suggesting no evidence of fungal multiplication. The results allowed suggesting the innocuity of B. bassiana LF14 when was administered intranasal to Mus musculus (NMRI strain).

Key words: Beauveria bassiana, pathogenicity, mice, infectivity

\section{INTRODUCCIÓN}

La enfermedad de Chagas o tripanosomiasis americana es considerada como una de las más importantes enfermedades metaxénicas de origen parasitario en Latinoamérica, estimándose que afecta alrededor de 8 millones de habitantes de 21 países (Rassi et al., 20010; WHO, 2012). Tiene una mayor prevalencia en asentamientos rurales de bajos recursos socio-económicos, donde el contacto con los vectores triatominos es más frecuente, siendo considerada como la enfermedad parasitaria que genera el mayor impacto socio-económico en Latinoamérica (WHO, 2012).

La aspersión en las casas con insecticidas de origen químico ha representado una medida práctica y efectiva de control a corto plazo contra los vectores triatominos; no obstante, su uso constante representa un peligro potencial para la salud del humano y los demás integrantes de la biósfera en general, induciendo, asimismo, mecanismos de resistencia en los vectores. Además, es una práctica inviable contra las poblaciones triatominas de hábitos peridomésticos o en zonas de selva (Luz et al., 2004; Forlani et al., 2011; WHO, 2012). Aunado a esto, se deben tener presentes los elevados costos de estos programas (Slinninger et al., 2003; WHO, 2012). Esto implica la necesidad de desarrollar es- trategias de control con enfoques más biorracionales, dentro de un programa de Manejo Integral de Plagas (MIP), en los que se incluyen microorganismos tales como los hongos entomopatógenos (Luz et al., 2004; Cazorla, 2011; Forlani et al., 2011).

Beauveria bassiana (Ascomycota: Hypocreales) es uno de los hongos entomopatógenos que ha mostrado una alta eficacia, tanto a nivel experimental como de campo, contra varias especies triatominas de importancia epidemiológica (Luz et al., 2004; Cazorla 2011; Forlani et al., 2011). Su efectividad depende de la capacidad de las conidias sobre los insectos muertos de esparcirse a través de las colonias de insectos (autodiseminación o transmisión fúngica horizontal) (Luz et al., 1999; Forlani et al., 2011). De aquí que, especialmente en ambientes cerrados como las habitaciones de las casas, la concentración de conidias en el aire va a depender del número de insectos infestados y de la humedad, entre otras condiciones (Luz et al., 1999; Ward et al., 2000).

Como consecuencia de las grandes cantidades de conidias y fragmentos fúngicos que pueden llegar a estar aerolizados, es inevitable que sean parte del aire que respiran los seres vivos, incluyendo el humano. Aunque los hongos entomopatógenos se consideran inocuos para los mamíferos (Zimmermann, 2007), la exposición a estos microorganismos 
biocontroladores se encuentra asociada a la aparición de síntomas respiratorios, tanto alérgicos como no alérgicos y daños a la función pulmonar (Douwes et al., 2003). En el caso particular de B. bassiana, se le ha aislado en el líquido pleural de un paciente con adenocarcinoma pulmonar y en muestras de esputos (Madsen et al., 2007).

Desde hace más de una década, el equipo multidisciplinario de investigación del presente estudio viene trabajando con hongos entomopatógenos de elevada virulencia contra especies triatominas de importancia epidemiológica y adaptados a las condiciones ecológicas de las zonas endémicas chagásicas de Venezuela (Cazorla, 2011). En este sentido, B. bassiana LF14 ha exhibido a nivel de laboratorio y especialmente en soluciones aceitosas, una elevada patogenicidad y virulencia hacia Rhodnius prolixus y Triatoma maculata (Cazorla, 2011), principales vectores de la enfermedad de Chagas en Venezuela (Feliciangeli, 2009).

Ante el eventual rociamiento de soluciones de conidias de B. bassiana LF14 en zonas pobladas, se hace necesario garantizar su inocuidad para los integrantes de la biósfera. En este sentido, se ha ensayado con éxito su bioseguridad por las vías dérmica (Acosta et al., 2011) y oral (Acosta et al., 2012) en el modelo ratón. El presente trabajo es la continuidad de estos bioensayos, al evaluar los efectos patogénicos por vía inhalatoria o respiratoria del aislamiento $B$. bassiana LF14 en ratones albinos (NMRI).

\section{MATERIALES Y Métodos}

Los ensayos se llevaron a cabo en el Laboratorio de Entomología, Parasitología y Medicina Tropical (LEPAMET) y en el bioterio de la Unidad de Parasitología y Medicina Tropical (UNIMETROPA) del Centro de Investigaciones Biomédicas (CIB), Campus Borregales, de la Universidad Nacional Experimental «Francisco de Miranda» (UNEFM), Coro, estado Falcón, Venezuela.
Se utilizó parte del diseño experimental previsto en el nivel I de evaluación toxicológica de los agentes microbianos para el control de plagas de la Agencia de Protección Ambiental (Environmental Protection Agency (EPA) de los Estados Unidos de América (US-EPA, 1996). Asimismo, se cumplieron las normas internacionales y nacionales de bioética y bioseguridad de las buenas prácticas de laboratorio, y en el cuidado y uso de animales de laboratorio (FONACIT, 2008) El presente protocolo de investigación fue aprobado por el Comité de Bioética de la UNEFM el 31 de enero de 2008 (Acosta $e t$ al., 2011).

\section{Animales de Experimentación}

Se usaron 48 ratones albinos heterocigotos NMRI (Mus musculus), de 6 a 7 semanas de edad, cuyos pesos no excedieron del $20 \%$ del peso promedio para cada sexo. Se trabajó con 12 ratones por sexo y grupo experimental (controles e inoculados). Los animales y su cuidado eran de calidad higiénico sanitarias conforme las normas de cuidado y uso de animales de laboratorio (FONACIT, 2008). Se colocaron tres animales por jaula de uso habitual en el bioterio. El alimento (ratarina: Protinal ${ }^{\circledR}$, Valencia, Venezuela) y el agua se esterilizaron por vapor húmedo en autoclave y fue suministrado $a d$ libitum. Los animales se mantuvieron en ciclo de luz-oscuridad de 12-12 horas (Acosta et al., 2011).

\section{Cultivo Fúngico y Suspensión de Conidias}

Las suspensiones de conidias se realizaron con el aislamiento LF14 de Beauveria bassiana, de la micoteca del Laboratorio de Fitopatología, Núcleo Universitario «Rafael Rangel» (NURR), Universidad de Los Andes (ULA), Trujillo, estado Trujillo, Venezuela (Fig. 1). Este fue aislado en 1992 a partir de un cadáver esporulado de insecto coleóptero no identificado recolectado en la población de Monay, estado Trujillo, región andino-venezolana. Las conidias se obtuvie- 


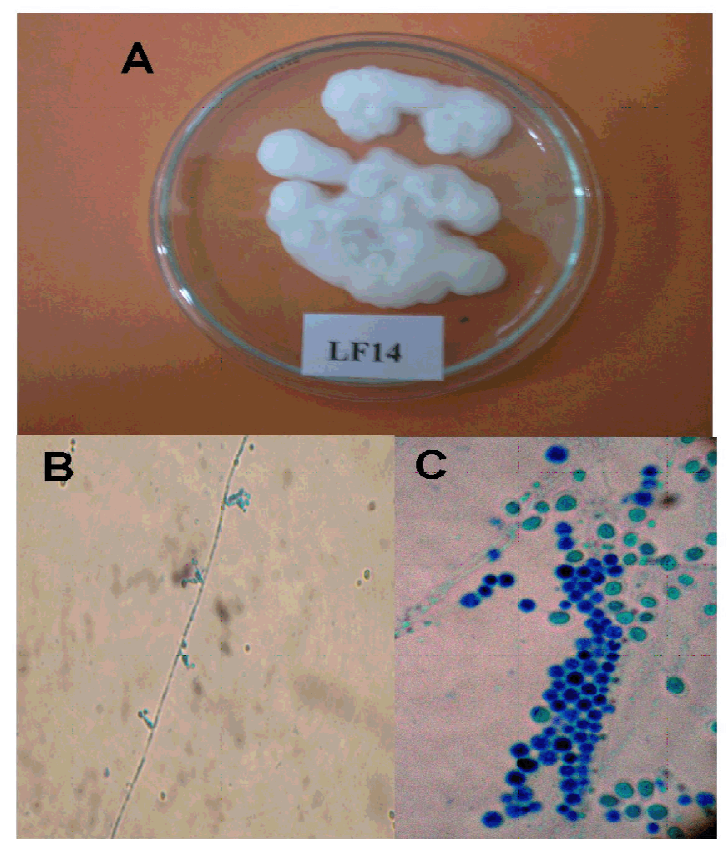

Figura 1. Morfología macroscópica en medio Sabouraud (A) y microscópica (B: conidióforos, células conidiógenas. C: conidias) (Azul de lactofenol, 400x) del aislamiento Beauveria bassiana LF14

ron raspando, mediante asa de platino, la superficie de cultivos esporulados de 15 días crecidos sobre medio sólido Lactrimel-agar (25 g glucosa, $10 \mathrm{~g}$ peptona, $20 \mathrm{~g}$ agar y 1000 ml agua destilada) (Borelli, 1962) y mantenidos en cámara de ambientación (Biotronette ${ }^{\circledR}$ Mark II, modelo 845, Lab Line Instruments, Illinois, EEUU), bajo condiciones de saturación de humedad relativa $(>90 \%)$ y a $26^{\circ} \mathrm{C}$ (Cazorla et al., 2007; Acosta et al., 2011).

El material fúngico cosechado se suspendió en agua destilada estéril y se filtró a través de gasa para separar el micelio de las conidias. Luego fue ajustado mediante cámara de Neubauer (hemocitómetro) (Neubauer-improved, Marienfeld, Alemania), a la concentración de $1 \times 10^{9}$ conidias $/ \mathrm{ml}$ (Goettel e Inglis, 1997; Cazorla et al., 2007) y utilizado de inmediato.

\section{Procedimiento de Infección}

Los animales se expusieron por instilación intranasal a una sola dosis de $0.3 \mathrm{ml} / \mathrm{kg}$ de una suspensión de $1 \times 10^{9}$ conidias $/ \mathrm{ml}$ del aislamiento LF14 de B. bassiana, utilizando un gotero nasal comercial (Fisiolin ${ }^{\circledR}$ ), donde se sustituyó el contenido comercial por la suspensión de conidias. Además, fue necesario colocar una aguja de inyectadora de insulina para facilitar la aplicación en las fosas nasales. Al grupo control se le aplicó el mismo volumen de gotas con agua destilada estéril.

Posteriormente, y por 14 días, se realizaron exámenes clínicos y de comportamiento, control diario de peso, mortalidad y estudio micológico (aclaramiento) por aspiración intranasal de secreción mucosa con pera de aspiración nasal. En los exámenes clínicos 


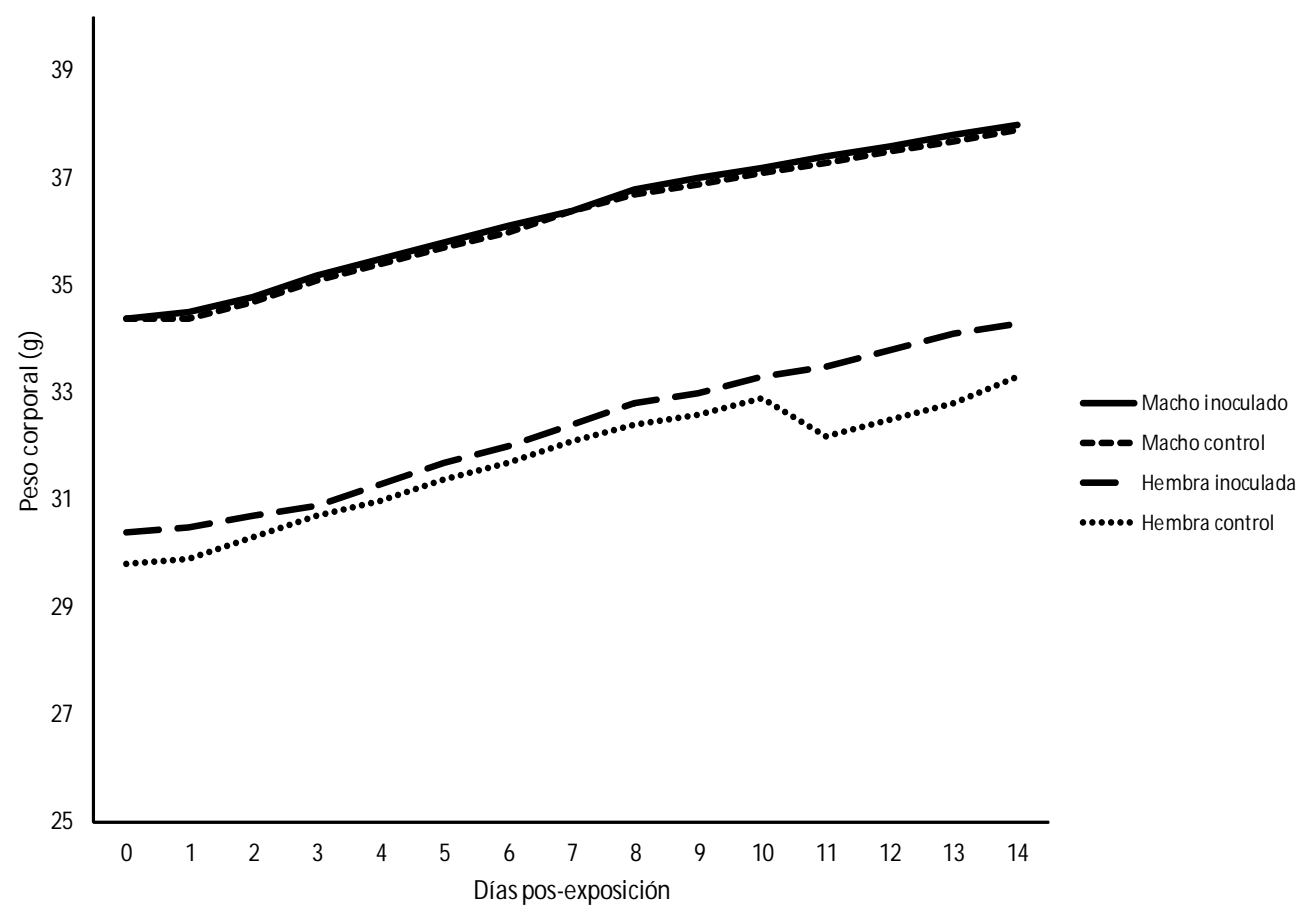

Figura 2. Peso promedio corporal (g) de ratones albinos durante los 14 días pos-exposición por vía inhalatoria a $1 \times 10^{9}$ conidias/ml del aislamiento LF14 Beauveria bassiana (12 ratones por grupo experimental)

se evaluó la presencia de signos de irritación en piel y pelo, ojos y mucosas, evaluación general de sistemas y patrones de conducta o comportamiento calificado como normalactivo, sensible, pasivo, agresivo (Tapias y Dussán, 2000); observándose, además, posibles efectos como convulsiones, diarrea, letargo, salivación, somnolencia y coma (Acosta et al., 2011).

Para el estudio micológico, las muestras de secreción se colocaron sobre láminas portaobjetos, se colorearon con azul de algodón, cubriéndose con laminilla cubreobjeto para observarse bajo microscopio fotónico (Axiostar Plus, Carl Zeiss, Alemania) (Cazorla et al., 2007). El cultivo de la muestra nasal se hizo en medio sólido (Sabouraud) por rastrilleo y las placas se incubaron por 714 días a $26^{\circ} \mathrm{C}$ y más de $90 \%$ de humedad relativa en cámara de ambientación (Cazorla et al., 2007). A las 24, 48 y 72 horas, se esti- mó el número de conidias germinadas (\%) (Cazorla et al., 2007). Una vez concluida la incubación, se cuantificó la esporulación (conidias/ml) mediante hemocitómetro (Goettel e Inglis 1997; Cazorla et al., 2007).

\section{Estudio Histopatológico}

Se practicó una inspección anatomo-patológica con una necropsia completa, observándose las características de órganos y sistemas en todos los ratones (Acosta et al., 2011). El sacrificio se hizo mediante anestesia con éter dietílico y dislocación cervical, en 6 a 9 animales por grupo experimental y sexo a los 3, 7 y 14 días después de la inoculación fúngica. Se tomaron muestras de piel, riñones, hígado, bazo, pulmones, tráquea, estómago, intestinos, corazón (Acosta et al., 2011), para estudio histopatológico y micológico directo y en cultivo en medio sólido, como ha sido previamente descrito. 


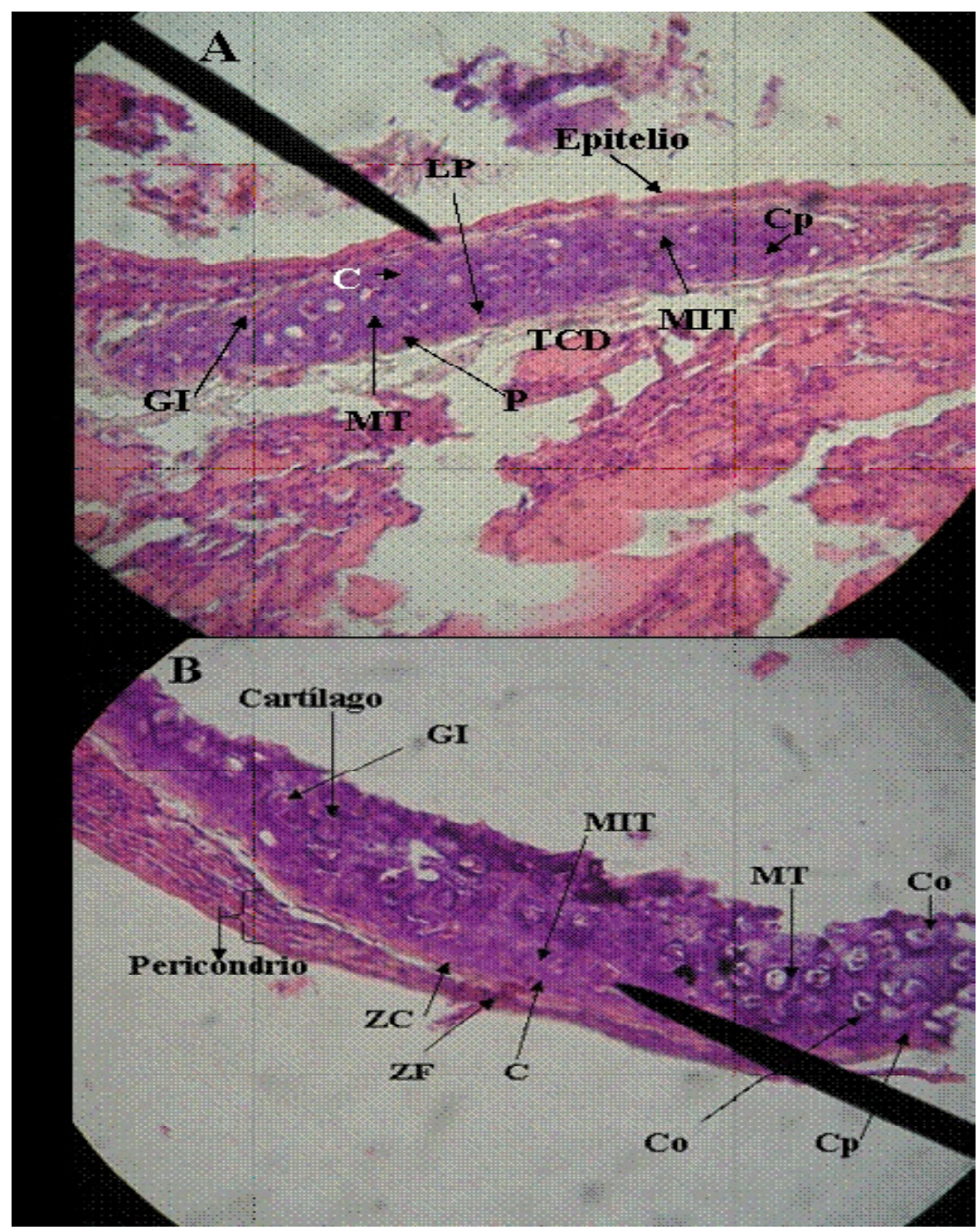

Figura 3. Sección de tráquea de ratones albinos: controles (A) y expuestos a Beauveria bassiana LF14 (B). Nótese ausencia daños histológicos discernibles e infiltrados inflamatorios (H-E, 400x). LP (lámina propia); TCD (tejido conjuntivo denso); $P$ (pericondrio); ZF (zona fibrosa); ZC (zona condrógena); $C$ (condroblastos); Co (condrocitos); Cp (condroplastos); GI (grupos isógenos); MT (matriz territorial); MIT (matriz inter-territorial)

En el estudio histopatológico, las muestras se fijaron en formaldehído al $10 \%$ y se incluyeron en parafina. Se hicieron cortes de 5-7 $\mu \mathrm{m}$ con micrótomo de rotación manual (Reichert Jung HN-40, Mannheim, Alemania), se colorearon con hematoxilina-eosina (H-E) o azul de toluidina, y se evaluaron y fotografiaron (Olympus, Fe-120, Olympus Imaging Corp., Japón) bajo un microscopio fotónico (Axiostar Plus, Carl Zeiss, Alemania) (Acosta et al., 2011).

\section{Análisis Estadístico}

La significancia estadística de las diferencias entre las medias de los pesos y esporulación (conidias/ml) se determinó mediante análisis de varianza de una vía y de comparación múltiple Student-NewmanKeusl (SNK). Los datos se analizaron mediante paquete estadístico MINITAB v. 13.20 (Minitab Inc. 2000). 


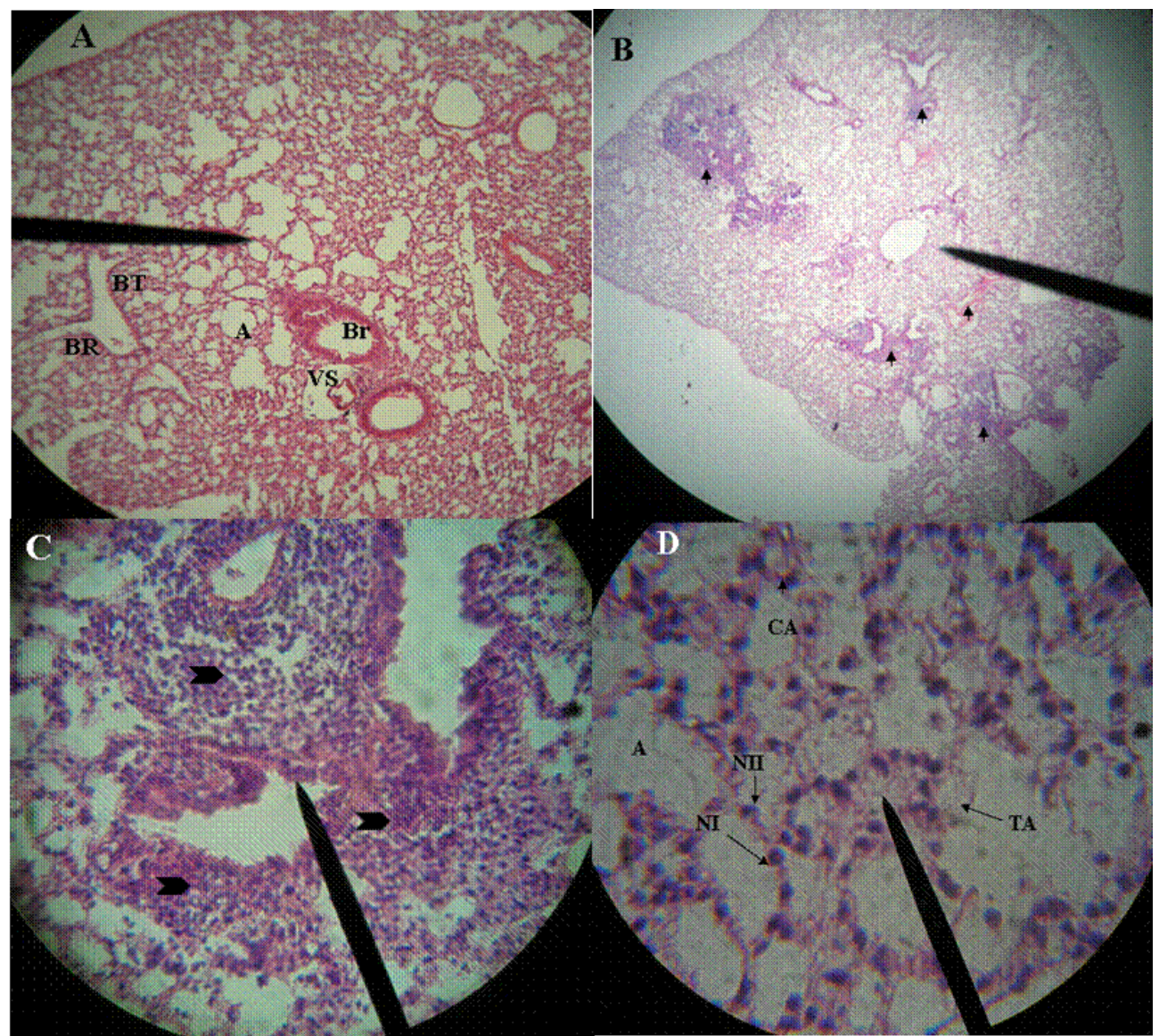

Figura 4. Sección de pulmón de ratones albinos controles (A) y expuestos a Beauveria bassiana LF14 (B) (400x), (C) (50x), D (1000x). Obsérvese pequeño infiltrado perivascular y peribronquial (flechas), con predominio de linfocitos y sin alteración anatómica de los espacios y tabiques alveolares (H\&E). BR (bronquiolo); bronquiolo terminal (BT); bronquiolo respiratorio $(B R)$; sacos alveolares $(A)$; tabiques interalveolares (TA); capilares alveolares (CA); VS (vaso sanguíneo); NI (neumocisto tipo I); NII (neumocisto tipo II)

\section{Resultados y Discusión}

No hubo registros de mortalidad ni alteraciones discernibles en los signos clínicos evaluados, observándose hábitos y comportamiento de tipo activo-normal, típicos para la especie (Tapias y Dussán, 2000).
En el análisis de la dinámica del peso promedio corporal, esta variable se incrementó en forma significativa en todos los grupos (machos y hembras inoculados y en los controles). Los machos presentaron mayores pesos que las hembras, tanto al inicio como al final del periodo de observación ( $\mathrm{F}=46,9$; $\mathrm{p}<0.0000$ ); sin embargo, no hubo diferencia 
estadística por sexo entre los animales inoculados y aquellos no expuestos a las conidias fúngicas (Fig. 2).

El análisis micológico de las muestras nasales durante los 14 días del ensayo, tanto por examen microscópico directo o cultivo en medio sólido, no reveló la presencia de la conidias de LF14 B. bassiana. Sin embargo, el estudio histopatológico reveló la presencia de conidias no germinadas en muestras del tracto respiratorio.

En el análisis de recuperación de conidias de animales (infectividad) con el cultivo de los órganos solo se obtuvieron resultados positivos (promedio de conidias $/ \mathrm{ml}$ \pm desviación estándar) en la necropsia realizada al tercer día de la exposición en pulmón $\left(\mathrm{n}=3\right.$ por sexo; machos $=7.5 \times 10^{5} \pm 1.1 \times 10^{4}$; hembras $\left.=7.8 \times 10^{5} \pm 2.1 \times 10^{4}\right)$ y tráquea $(\mathrm{n}=3$ por sexo; machos $=2.7 \times 10^{5} \pm 3.3 \times 10^{4}$; hembras $=3.1 \times 10^{5} \pm 1.5 \times 10^{4}$ ); sin embargo, no hubo diferencia estadística entre sexos.

En el examen macroscópico externo, durante la necropsia, no se evidenciaron hallazgos patológicos ni diferencias entre los grupos de ratones expuestos a las conidias fúngicas y los grupos controles. Similarmente, tampoco se detectaron alteraciones patológicas macroscópicas en las cavidades torácica y abdominal y sus órganos.

En el estudio histopatológico, no se evidenciaron alteraciones estructurales de consideración en los tejidos en órganos de los ratones inoculados. De una manera global, no se observaron infiltrados de macrófagos, neutrófilos y eosinófilos como parte de un proceso inflamatorio agudo, así como tampoco conidias germinadas ni evidencias fúngicas o histopatológicas de la multiplicación del aislamiento LF14 (Figs. 3 y 4). Sin embargo, se observaron leves cambios inflamatorios caracterizados por la presencia de un mínimo infiltrado perivascular y peribronquial en los cortes de pulmones (Fig. 4).
Uno de los requisitos indispensables para registrar y comercializar un bioplaguicida ante los entes gubernamentales reguladores es la demostración de que no infecta al humano ni a los animales domésticos, y que debe ser seguro para los seres vivos integrantes del ecosistema (Toriello, 2003). La exposición inhalatoria es una de las pruebas toxicológicas del nivel I que se requieren para probar la seguridad de un producto fúngico sin formular (Jaronski et al., 2003; Toriello, 2003).

En el presente trabajo quedó demostrado la inocuidad en ratones NMRI del aislamiento B. bassiana LF14 administrado intranasalmente con $1 \times 10^{9}$ conidias $/ \mathrm{ml}$. Este resultado coincide con los hallazgos de varios autores que trabajaron con ratones y otras especies de vertebrados empleando otras cepas de B. bassiana y otras especies de ascomicetos (El-Kadi et al., 1983; Tapias y Dussán, 2000; Mancebo et al., 2005, 2009; US-EPA, 2006; Zimmermann, 2007).

En forma similar a otros ensayos de evaluación del aislamiento $B$. bassiana LF14 por vía dérmica (Acosta et al., 2011) y oral (Acosta et al., 2012), el incremento de peso estuvo dentro de los rangos aceptados para individuos adultos de M. musculus (QuesadaDomínguez, 1997). No obstante, Mancebo et al. (2005) detectaron una disminución del peso en ratas cuando se les administró Paecilomyces lilacinus por vía intravenosa, lo que atribuyeron como parte del proceso patogénico del hongo. En lo que respecta a las diferencias entre sexos, se espera en $M$. musculus un mayor peso corporal en el macho (Quesada-Domínguez, 1997; Tapias y Dussán, 2000; Acosta et al., 2011, 2012).

En el estudio de aclaramiento no se detectaron cultivos positivos en tracto respiratorio, aunque en la evaluación anatomopatológica se pudo observar conidias viables en pulmón y tráquea en el tercer día pos-exposición, así como leves procesos inflamatorios con infiltrado perivascular y 
peribronquial, lo que pareciera sugerir que $B$. bassiana LF14 posee algún grado de inmunogenicidad. Sin embargo, no se encontró evidencia micológica ni histopatológica sobre la germinación y multiplicación del hongo, aún a las elevadas dosis inoculadas.

Se observaron algunos contrastes al comparar estos resultados con otras cepas de $B$. bassiana y hongos entomopatógenos. Así, Tapias y Dussán (2000), trabajando con tres cepas de $B$. bassiana inoculadas intratraquealmente en ratones $\mathrm{BALB} / \mathrm{c}$ y Mancebo et al. (2005, 2009) inoculando intranasalmente conidias del hongo entomopatógeno $P$. lilacinus y la cepa $B$. bassiana 9205 en ratas, no llegaron a observar alteraciones a nivel del árbol respiratorio ni recuperaron las conidias; mientras que Ward et al. (1998, 2000) encontraron respuestas inmunológicas, inflamatorias, histopatológicas y fisiológicas en ratones BALB/c expuestos a antígenos crudos de Metarrhizium anisopliae en sus vías respiratorias. Asimismo, Song (1989) encontró neumonía intersticial crónica, con neumonitis descamativa, fibrosis y edema pulmonar en el $79 \%$ de ratas y ratones expuestas por 18 meses a conidias de B. bassiana.

Es conocido que las variaciones de temperatura ocasionan una amplia variedad de daños irreversibles en las células fúngicas (Ulusu y Tezcan, 2001; Rangel et al. 2005). Temperaturas mayores de $35{ }^{\circ} \mathrm{C}$ en los vertebrados homeotermos, como la de los ratones $\left(37^{\circ} \mathrm{C}\right)$ (Quesada-Domínguez, 1997), es uno de los factores que interviene en la falta de patogenicidad de los hongos entomopatógenos, ya que estos microorganismos son termosensibles (Hallsworth y Magan, 1999; Rijo et al., 2002). El aislamiento $B$. bassiana LF14 no logra germinar ni desarrollarse in vitro entre $35 \mathrm{y}$ $38^{\circ} \mathrm{C}$ (Cazorla et al., 2007); sin embargo, se han reportado casos humanos, mayormente en inmucomprometidos con procesos infecciosos debidos a Beauveria (Henke et al., 2002) y por otros hongos entomopatógenos, como M. anisopliae (Revankar et al., 1999) y P. lilacinus (Prado y Borelli, 1993). Lo llamativo de estos hallazgos es que estos hongos no proliferan en cultivos in vitro a $37^{\circ} \mathrm{C}$, donde similar comportamiento se ha observado en otros hongos oportunistas (Alternaria sp, Ulocladium sp, Fusarium sp, Sporothrix sp y Engyodontium sp) (Shimazu, 2004). Por lo tanto, la termotolerancia in vivo para estos taxones fúngicos puede ser diferente a la detectada in vitro.

Aunque los hongos entomopatógenos, incluyendo B. bassiana, no suelen ocasionar daños considerables a nivel respiratorio en el humano; no obstante, poseen sustancias que son potenciales alergenos; además, se les encuentra presentes en el aire y otros sustratos, por lo que podrían ocasionar problemas patológicos en las vías respiratorias, especialmente en individuos inmucomprometidos (Ward et al., 2000; Lackner et al., 2004), en uno de los cuales se ha reportado signos de alveolitis muy parecidos a las manifestaciones clínicas de hipersensibilidad tipo III y IV detectadas en ratones y ratas expuestas experimentalmente a conidias de $B$. bassiana (Song, 1989; Henke et al., 2002).

En este mismo sentido, estudios hechos con pruebas alérgicas han demostrado que existen índices de alergenicidad hacia extractos de B. bassiana (Beaumont et al., 1984, 1985; Westwood et al., 2005). Además, se han detectado alergias de tipo ocupacional debida a la exposición con hongos entomopatógenos (Green et al., 2009). Por otra parte, B. bassiana posee epitopes que son homólogos a otras especies fúngicas de mucha relevancia clínica (Alternaria sp, Penicillium sp, Candida sp, Aspergillus sp), especialmente en el desarrollo de reacciones alérgicas mediadas por $\operatorname{IgE}$ en individuos atópicos, de allí que existe la posibilidad que un individuo desarrolle una reacción alérgica a Beauveria, si previamente presentó sensibilidad hacia otra especie de hongo (Westwood et al., 2005). 


\section{Conclusiones}

El aislamiento $B$. bassiana LF14 se presenta inocuo por vía inhalatoria en $M u s$ musculus (NMRI).

\section{Literatura Citada}

1. Acosta M, Cazorla D, Eduarte G, Morales $P$. 2011. Patogenicidad dérmica de un aislamiento autóctono del hongo entomopatógeno Beauveria bassiana en ratones. Rev Científ FCV-LUZ 21: 477 483.

2. Acosta M, Cazorla D, Eduarte G, Morales P. 2012. Patogenicidad oral de un aislamiento autóctono del hongo entomopatógeno Beauveria bassiana en ratones (Mus musculus, cepa NMRI). Bol Mal Salud Amb 52: 79-86.

3. Beaumont $\mathrm{F}$, Kauffman $\mathrm{H}$, Sluiter $\mathrm{H}$, de Vries K. 1984. A volumetricaerobiologic study of seasonal fungus prevalence inside and outside dwellings of asthmatic patients living in northeast Netherlands. Ann Allergy 53: 486-492.

4. Beaumont F, Kauffman H, de Monchy J, Sluiter H, de Vries K. 1985. Volumetric aerobiological survey of conidial fungi in the North-East Netherlands. II. Comparison of aerobiological data and skin tests with mould extracts in an asthmatic population. Allergy 40: 181-186.

5. Borreli D. 1962. Medios caseros para micología. Arch Ven Trop Parasitol Méd 4: 301-310.

6. Cazorla D, Morales P, Acosta M. 2007. Efectos de gradientes térmicos, salinos y $\mathrm{pH}$ sobre la germinación in vitro de un aislado nativo de Beauveria bassiana (bálsamo) Vuillemin, patógeno para Rhodnius prolixus y Triatoma maculata. Rev Científ FCV-LUZ. 17: 627-631.

7. Cazorla D. 2011. El uso de hongos entomopatógenos para el control biorracional de Triatominae, vectores de la enfermedad de Chagas. Avances Cardiol 31: 333-352.

8. Douwes J, Thorne P, Pearce N, Heederik D. 2003. Bioaerosols health effects and exposure assessment: progress and prospects. Ann Occup Hyg. 47: 187-200. doi: 10.1093/annhyg/ meg032

9. El-Kadi M, Xará L, De Matos P, Da Rocha J, De Oliveira D. 1983. Effects of the entomopathogen Metarhizium anisopliae on guinea pigs and mice. Environ Entomol 12: 37-42. doi: 10.1093/ ee/12.1.37

10. Feliciangeli M. 2009. Control de la enfermedad de Chagas en Venezuela. Logros pasados y retos presentes. Interciencia 34: 393-399.

11. [FONACIT] Fondo Nacional de Ciencia y Tecnología. 2008. Código de bioética y bioseguridad [Internet]. Disponible en: http://www.ciens.ucv.ve:8080/generador/sites/biolanimlab/archivos/codigo_fonacit_2008.pdf

12. Forlani L, Pedrini N, Juárez M. 2011. Contribution of the horizontal transmission of the entomopathogenic fungus Beauveria bassiana to the overall performance of a fungal powder formulation against Triatoma infestans. Res Rep Trop Med 2: 135-140. doi: 10.2147/RRTM.S22961

13. Goettel M, Inglis G. 1997. Fungi: Hyphomycetes. En: Lacey L (ed). Manual of techniques in insect pathology. San Diego, California, EEUU: Academic Press. p 213-249.

14. Green B, Tovey E, Beezhold D, Perzanowski M, Acosta L, Divjan I, Chew G. 2009. Surveillance of fungal allergic sensitization using the fluorescent halogen immunoassay. J Mycol Med 19: 253-261. doi: 10.1016/j.mycmed.2009.10.003

15. Hallsworth J, Magan N. 1999. Water and temperature relations of growth of the entomogenous fungi Beauveria bassiana, Metarhizium anisopliae, and Paecilomyces farinosus. J Invertebr Pathol 74: 261-266. doi: 10.1006/ jipa.1999.4883 
16. Henke M, De Hoog G, Gross $U$, Zimmermann G, Kraemer D, Weig M. 2002. Human deep tissue infection with an entomopathogenic Beauveria species. J Clin Microbiol 40: 2698-2702.

17. Jaronski S, Goettel M, Lomer C. 2003. Regulatory requirements for ecotoxicological assessments of microbial insecticides - How relevant are they? En: Hokkanen H, Hajek A (eds). Environmental impacts of microbial insecticides. Holanda: Kluwer Academic Publishers. p 237-260.

18. Lackner A, Freudenschuss K, Buzina $W$, Stammberger $H$, Panzitt $T$, Schosteritsch S, Braun H. 2004. From when on can fungi be identified in nasal mucus of humans? Laryngorhinootologie 83: 117-121.

19. Luz C, Silva I, Cordeiro C, Tigano M. 1999. Sporulation of Beauveria bassiana on cadavers of Triatoma infestans after infection at different temperatures and doses of inoculum. $\mathbf{J}$ Invertebr Pathol 73: 223-225.

20. Luz C, Rocha L, Nery G Magalhaes B, Tigano M. 2004. Activity of oilformulated Beauveria bassiana against Triatoma sordida in peridomestic areas in Central Brazil. Mem Inst Oswaldo Cruz 99: 211-218. doi: /10.1590/S007402762004000200017

21. Madsen A, Hansen V, Meyling N, Eilenberg J. 2007. Human exposure to airborne fungi from genera used as biocontrol agents in plant production. Ann Agric Environ Med 14: 5-24.

22. Mancebo A, González F, González B, Riera L, Lugo S, Bada A, Aldana L, et al. 2005. Evaluación de la patogenicidad en ratas del Paecilomyces lilacinus LPL-01 utilizando vías diferentes de exposición. Rev Toxicol 22: 185190.

23. Mancebo A, González F, Aldana L, González Y, Lugo S, González B, Fuentes D, et al. 2009. Pathogenicity of the entomopathogenic fungus Beauveria bassiana 9205 in rats using different routes of exposition. Toxicol Environ Chem 91: 99-108. doi: 10.1080/ 02772240802010524

24. Prado A, Borelli D. 1993. Paecilomyces lilacinus: cepa polimorfa aislada de dermatitis pioverrugoide posttraumática. Derm Venez 31: 99-103.

25. Quesada-Domínguez A. 1997. Introducción al manejo de los animales de laboratorio: roedores y pequeñas especies. Mérida, Yucatán, México: Ed Universidad Autónoma de Yucatán. 151 p.

26. Rangel D, Braga G, Anderson A, Roberts D. 2005. Variability in conidial thermotolerance of Metarhizium anisopliae isolates from different geographic origins. J Invertebr Pathol 88: 116-125. doi: 10.1016/j.jip.2004.11.007

27. Rassi A Jr, Rassi A, Marin-Neto J. 2010. Chagas disease. Lancet 375 : 1388-1402. doi: 10.1016/S01406736(10)60061-X

28. Revankar S, Sutton D, Sanche S, Rao $J$, Zervos M, Dashtand F, Rinaldi $M$. 1999. Metarrhizium anisopliae as a cause of sinusitis in immunocompetent hosts. J Clin Microbiol 37: 195-198.

29. Rijo E, Luján M, Martínez J, Oliva J. 2002. Patogenicidad de los hongos Verticillium lecanii (Zimmermann) Viegas y Beauveria bassiana (Balm) Vuilleman a animales homeotermos. Rev CENIC Cien Biol 33: 33-35.

30. Shimazu M. 2004. Effects of temperature on growth of Beauveria bassiana F-263, a strain highly virulent to the Japanese pine sawyer, Monochamus alternatus, especially tolerance to high temperatures. App Entomol Zool 39: 469-475. doi: 10.1303/aez.2004.469

31. Slinninger P, Behle R, Jackson M, Schisler D. 2003. Discovery and development of biological agents to control pests. Neotrop Entomol 32: 183-195. doi: 10.1590/S1519-566X2003000200001

32. Song J. 1989. Experimental study on farmer's lung-like lesions caused by Beauveria bassiana. Zhonghua Bing Li Xue Za Zhi 18: 111-114. 
33. Tapias S, Dussán J. 2000. Evaluación del grado de seguridad del hongo Beauveria bassiana utilizado para el control biológico de insectos plaga. Actual Biol 22: 17-27.

34. Toriello C. 2003. Bioseguridad de Metarhizium anisopliae (Metschnikoff) Sorokin (Hyphomycete). Vedalia 9-10: 107-113.

35. Ulusu N, Tezcan E. 2001. Cold shock proteins. Turk J Med Sci 31: 283-290.

36. [US-EPA] United State Enviromental Protection Agency. 1996. Microbial pesticide test guidelines OPPTS 885.3000. Background-mammalian toxicity/pathogenicity/infectivity. EPA 712-C-96-314. [Internet]. Disponible en: ht tp://www.regulations.gov/ \#!documentDetail;D=EPA-HQ-OPPT2009-0159-0018

37. Ward M, Sailstad D, Selgrade M. 1998. Allergic responses to the biopesticide Metarhizium anisopliae in BALB:c mice. Toxicol Sci 45: 195-203.
38. Ward M, Madison S, Sailstad D, Gavett S, Selgrade M. 2000. Allergentriggered airway hyperresponsiveness and lung pathology in mice sensitized with the biopesticide Metarhizium anisopliae. Toxicology 143: 141-154. doi: 10.1016/S0300-483X(99)00166-3

39. Westwood G, Huang S, Keyhani N. 2005. Allergens of the entomopathogenic fungus Beauveria bassiana. Clin Mol Allergy 3:1. doi: 10.1186/14767961-3-1

40. [WHO] World Health Organization. 2012. Research priorities for Chagas disease, human African trypanosomiasis and leishmaniasis. Ginebra, Suiza: WHO Technical report series $\mathrm{N}^{\circ} .975 .100 \mathrm{p}$.

41. Zimmermann G. 2007. Review on safety of the entomopathogenic fungi Beauveria bassiana and Beauveria brongniartii. Biocontrol Sci Technol 17: 553-596. doi: 10.1080/09583150701309006 